\title{
Aplicación web e-learning multiplataforma para recolección de datos de usuarios y retroalimentación automática basada en técnicas estadísticas
}

\author{
Samuel Montiel Santos ${ }^{1}$, Luis Ángel Reyes Hernández ${ }^{1}$, Asdrúbal López-Chau², \\ Beatriz Alejandra Olivares Zepahua ${ }^{1}$, Celia Romero Torres ${ }^{1}$ \\ ${ }^{1}$ División de Estudios de Posgrado e Investigación, Instituto Tecnológico de Orizaba, \\ Orizaba, Veracruz, México \\ ${ }^{2}$ Universidad Autónoma del Estado de México, \\ Zumpango, Estado de México, México \\ smontiels@outlook.com,1_r_h01@hotmail.com, alchau@uaemex.mx, \\ bolivares@ito-depi.edu.mx, cromerotorres@ hotmail.com
}

\begin{abstract}
Resumen. Las generaciones actuales de jóvenes conviven en entornos donde es común la presencia de dispositivos electrónicos conectados a Internet. Entre las diferentes aplicaciones disponibles se encuentran las de e-learning. Sin embargo, un aspecto poco abordado, es la utilización de técnicas estadísticas, aprendizaje automático y minería de datos para retroalimentar tanto a discentes y profesores para mejorar su desempeño académico o su función docente. En este artículo, se propone una arquitectura escalable para aplicaciones e-learning para dispositivos móviles, con capacidad de ofrecer retroalimentación automática a los usuarios basadas en la interacción con el sistema y las calificaciones obtenidas. La arquitectura propuesta permite incorporar diversas técnicas, incluyendo las de inteligencia artificial, para mejorar la calidad de la retroalimentación. Para demostrar la viabilidad del enfoque, se desarrolló un prototipo de aplicación que permite identificar posibles dificultades académicas y emitir recomendaciones a discentes de nivel licenciatura usando técnicas estadísticas.
\end{abstract}

Palabras clave: aplicación móvil, e-learning, arquitectura.

\section{Cross-platform E-learning Application for User Data Collection and Feedback}

Abstract. Current generations of young people coexist in environments where electronic devices connected to the Internet are common. Among the different 
applications available are e-learning. However, one aspect that is little discussed in the literature is the use of statistical techniques, machine learning and data mining to feed both students and teachers to improve their academic performance or their teaching function. In this article, we propose a scalable architecture for e-learning applications for mobile devices, capable of offering automatic feedback to users based on interaction with the system, and the scores obtained. The proposed architecture allows to incorporate diverse techniques, including those of artificial intelligence, to improve the quality of the feedback. To demonstrate the feasibility of the approach, a prototype application was developed to identify possible academic difficulties and to issue recommendations to undergraduate students using statistical techniques.

Keywords: mobile app, e-learning, architecture.

\section{Introducción}

Hoy en día existen una gran cantidad de aplicaciones móviles y cada día se suman muchas más, donde gran parte se dirige hacia el público joven, dado que son los mayores consumidores de estos contenidos. Por esta razón, se crean en su mayoría aplicaciones enfocadas a temas de entretenimiento y diversión. Aunado a lo anterior, una persona promedio gasta mensualmente 35 horas en el uso de dispositivos móviles y $9 \%$ de esas horas son para videojuegos y actividades no propias del estudio [1], por lo que no existen suficientes canales que ofrezcan apoyo a la difusión o mejora del aprendizaje. Debido a la inclusión de los dispositivos móviles en la actualidad, una gran cantidad de personas tienen la posibilidad de acceso a ellos, por lo que actualmente se cuentan con 2,600 millones de suscriptores de este tipo de medios electrónicos [2]. Aprovechando lo anterior, en este artículo se propone la utilización de teléfonos inteligentes con fines académicos. Específicamente, se propone la arquitectura de una aplicación e-learning para retroalimentar a usuarios, tanto docentes como discentes.

La arquitectura escalable presentada en este artículo se basa en el concepto de sistemas e-learning, que hacen uso de las redes y las tecnologías multimedia, permitiendo el acceso al conocimiento y a los recursos remotos para el desarrollo de toda la sociedad, por lo que se centra en la importancia de la utilización de aplicaciones móviles en el ámbito de la educación a nivel licenciatura, y en la necesidad de desarrollar nuevas herramientas que permitan la transmisión de conocimientos de manera diferente, así como también sirvan de apoyo a los métodos de enseñanza que se utilizan actualmente.

En este sentido, una parte de la arquitectura propuesta contempla una aplicación móvil e-learning, que sea utilizada en grupos de discentes guiados por su profesor para la aplicación de evaluaciones de ciertos temas. Esto con el fin de que los jóvenes que se encuentran cursando actualmente el grado de licenciatura se apoyen en herramientas que sirvan de soporte para un mejoramiento continuo a comparación con las generaciones anteriores que estaban en condiciones diferentes en tecnología, dado que hoy en día las 
personas se encuentran mejor comunicadas y comparten constantemente gran cantidad de información.

La arquitectura mostrada se basa en el patrón arquitectónico de software Modelo-VistaControlador el cual separa los datos y la lógica de negocio de la interfaz de usuario. Para que de esta manera se aprecie de manera fácil y sencilla la forma de interacción entre los distintos módulos solicitando peticiones y recibiendo respuestas para cada uno de ellos. De igual forma la arquitectura cuenta con un módulo basado en machine-learning, el cual es una disciplina científica del ámbito de la Inteligencia Artificial que crea sistemas que aprenden automáticamente, en este contexto quiere decir identificar ciertos patrones a través de la información disponible, este módulo se agregó para el análisis de datos y la retroalimentación de los discentes basado en las calificaciones obtenidas en sus evaluaciones.

Para una mejor explicación del objeto de estudio, el presente artículo se encuentra estructurado de la siguiente forma: La Sección 2 muestra el estado del arte de los artículos relacionados con el tema de investigación, la Sección 3 describe la arquitectura de la aplicación, la Sección 4 muestra los resultados y finalmente, las conclusiones y el trabajo a futuro se describen en la Sección 5.

\section{Trabajos relacionados}

Las nuevas tecnologías y la globalización brindan un cambio rápido en todos los aspectos de la vida humana. Entre ellos el incremento del interés por los dispositivos móviles y su uso para la enseñanza y la investigación. Es por ello que M. Isidora et al. [3] propusieron utilizar paquetes de software para examinar el modo de actuar que tienen los discentes sobre este tipo de nuevas técnicas de enseñanza, ofreciendo mejoras durante el proceso de aprendizaje y haciendo atractivo el modo de enseñanza, tratando de obtener satisfacción durante el proceso de educación.

Hoy en día existen varios sistemas operativos móviles que son muy populares a nivel mundial, tales como Android e iOS, entre otros más, y esto representa un inconveniente para el desarrollo de aplicaciones debido a los diferentes lenguajes de programación y a sus distintos modos de funcionamientos. Es por ello que D. Haryadi [4] desarrolló una aplicación híbrida (diferentes sistemas operativos) e-learning que ayuda a los discentes a obtener información de ciertos cursos que se imparten, esta aplicación se ejecuta dentro de un contenedor nativo en cada dispositivo móvil, utilizando HTML5, JavaScript y CSS.

En este trabajo se desarrolló una aplicación móvil e-learning que permitiera familiarizarse con el pensamiento y lenguaje legal de una manera eficiente, la cual se basó en un escenario hipotético, haciendo que los discentes completaran ciertas tareas y lograran metas de aprendizaje utilizando análisis, investigación jurídica y casos de estrategia, $\mathrm{V}$. Bouki et al. [5] describieron que la gamification (ludificación) fomenta la motivación del 
aprendizaje, esto mediante un enfoque educativo para motivar a los discentes a aprender mediante el uso de videojuegos en ambientes de enseñanza.

J. López, A. Cerezo y J. Menéndez [6] desarrollaron una aplicación experimental utilizando Java para dispositivos con sistema operativo móvil Android para la preparación de un examen para obtener la acreditación para guía turístico adaptando los contenidos pertinentes a dicha aplicación, como una simulación del examen para facilitar el conocimiento.

L. Flores, D. Almario y J. López [7] desarrollaron una herramienta que ayude a los discentes a entender cómo funcionan los sistemas dinámicos. Por lo que se diseñó y desarrolló una aplicación para el sistema operativo móvil Android que representa matemáticamente este sistema, y para ello se implementó un modelo diferencial de ecuaciones utilizando el método numérico de Runge-Kutta.

M. Chacon y C. Garita [8] desarrollaron una aplicación que permitió abordar el tema de m-learning como una nueva modalidad de aprendizaje y tomar ventaja de la creciente utilización de celulares inteligentes y tabletas electrónicas. En particular, la aplicación representó la primera aplicación dirigida a discentes con el objetivo de brindar acceso a la información general sobre cursos.

Los dispositivos móviles tales como teléfonos, tabletas y laptops, actualmente se venden a un precio económico y se obtienen de varias maneras accesibles por lo que J. Hou, K. Zhang X. Yang [9] exploraron integrar la tecnología m-learning en los cursos en línea que se utilizan en la Escuela de Educación Continua de la Universidad de Pekín lo que proporcionó a los discentes un modo de aprendizaje combinado, en donde ellos pudieron acceder a cualquiera de los cursos disponibles a través de laptop, teléfonos móviles o tabletas sin ningún problema.

Se desarrolló una aplicación móvil llamada CyberAware por parte de F. Giannakas et al. [10] destinada a la educación y la conciencia de la seguridad cibernética, fomentando factores educativos, con el objetivo de familiarizar a los discentes con las tecnologías de seguridad cibernética fundamentales que se necesitan al estar conectados a Internet y mantener la protección continua contra el malware, los ataques cibernéticos, y el spam.

A. Sarasa y L. Sierra [11] desarrollaron una aplicación móvil Android que permitió a los discentes interactuar con todas las actividades disponibles en la institución a través de la aplicación. Además el usuario profesor era el responsable de configurar y gestionar las actividades propuestas y aceptar si así lo deseaba a los discentes interesados en los cursos que imparte y de esta manera facilitar la gestión necesaria para todas aquellas actividades realizadas en todos los niveles de la Universidad.

Reconociendo el enorme potencial de las aplicaciones de Android, H. Lye y S. Yean [12] desarrollaron una aplicación móvil para simular y comparar la implementación de los tres métodos de Black Scholes sobre una plataforma móvil, con el objetivo de animar a los discentes de ingeniería financiera para explorar el mundo de los Modelos de Black-Scholes y aprender más sobre el riesgo de los instrumentos financieros. 
La Universidad de Hong Kong cuenta con un LMS (Learning Management System, Sistema de gestión del aprendizaje) que se accede de manera Web, pero también desarrolló una aplicación móvil denominada "HKU Space Soul" con el objetivo de trasladar a los discentes a una plataforma móvil, facilitando con esto la mejora continua del aprendizaje. Como resultado, el total de número de accesos al LMS vía aplicación móvil aumentó, mientras que el número de accesos desde el navegador Web disminuyó. Por lo que P. Hung, J. Lam y C. Wong en [13] mostraron la importancia de ofrecer nuevos servicios tecnológicos a los profesores y discentes, debido a que el uso de la tecnología educativa móvil mejora las experiencias de aprendizaje.

Hoy en día hay gran número de universidades trabajan en el desarrollo de laboratorios virtuales y remotos. Es por ello que S. Martin et al. en [14] desarrollaron una arquitectura para el apoyo a la creación de aplicaciones m-learning que consuman la información alojada en su LMS. Permitiendo a los discentes el acceso a los contenidos y servicios en cualquier momento, en cualquier lugar y en cualquier tipo de dispositivo. Como resultado final se logró generar una clasificación de los diferentes tipos de aplicaciones que proporcionan experiencia de aprendizaje y los detalles específicos necesarios del diseño del middleware propuesto.

En [15] se presentó un enfoque para evaluar y predecir el nivel de competencia del discente cuando se utiliza una determinada estrategia de enseñanza. Utilizando una de las técnicas del machine-learning, en particular, el uso de circuitos lógicos/redes para dicha predicción. El modelo matemático de los circuitos lógicos se utiliza para evaluar el nivel de competencia. Proponen también un método para sintetizar el Circuito lógico resultante que predice la efectividad del proceso de enseñanza cuando dos estrategias se combinan. Los resultados preliminares experimentales demuestran la escalabilidad esperada y la aplicabilidad del enfoque propuesto.

En [16] se propone un método de predicción de deserción para cursos de e-learning, basado en tres técnicas populares de machine-learning y datos detallados de los discentes. Las técnicas de aprendizaje mecánico utilizadas son las redes neuronales feed-forward, las máquinas vectoriales de apoyo y el conjunto probabilístico simplificado ARTMAP (Teoría de la Resonancia Adaptativa). El método se examinó en términos de precisión general, sensibilidad y precisión y sus resultados fueron altos, lo que indica que el esquema era preciso tanto en la identificación correcta de los abandonos como en evitar las clasificaciones erróneas más completas.

Por otra parte, en [17] se construyó un sistema de evaluación de la gestión de los discentes a través de la tecnología JSP (Java Server Pages), realizando un análisis en profundidad para el marco de evaluación reciente y el procesamiento de datos no estructurados y Datos semi-estructurados. Por otro lado, considerando la complejidad de la información para la gestión estudiantil y la subjetividad de las mediciones, se propuso un algoritmo basado en la Red Neural Artificial (ANN Artificial Neural Network) con el objetivo de construir un modelo integrado inteligente a través de la tecnología de minería 
de datos. Al evaluar un conjunto de datos de gestión recogidos de una escuela politécnica se mostró la efectividad del algoritmo propuesto.

En la Tabla 1 se muestra una breve comparación entre algunos trabajos antes descritos y este artículo.

Tabla 1. Análisis Comparativo del estado del arte.

\begin{tabular}{cccccc}
\hline Trabajo & Manejo de Estadística & A. Android & A. iOS & A. Web & G. Varios temas \\
\hline$[3]$ & Sí & No & No & No & Sí \\
{$[4]$} & No & Sí & Sí & Sí & No \\
{$[5]$} & No & No & Sí & No & No \\
{$[6]$} & No & Sí & No & No & No \\
{$[7]$} & No & Sí & No & No & No \\
{$[8]$} & No & Sí & No & No & Sí \\
{$[9]$} & No & No & No & No & Sí \\
{$[10]$} & Sí & Sí & Sí & Sí & No \\
{$[11]$} & Sí & Sí & No & Sí & Sí \\
{$[12]$} & No & Sí & No & No & No \\
{$[13]$} & No & No & Sí & Sí & Sí \\
{$[14]$} & No & No & No & No & Sí \\
\hline Propuesta & Sí & Sí & Sí & Sí & Sí \\
\hline
\end{tabular}

Como se logra apreciar en la Tabla 1, ningún artículo menciona la posibilidad de manejo de técnicas estadísticas para generar retroalimentación tanto a docentes como a discentes. Por lo que se concluye que se puede utilizar esa carencia a favor del proyecto ocupando un módulo Generador de Feedback (retroalimentación) para el análisis de patrones utilizando la información que se genere al utilizar dicho proyecto.

\section{Arquitectura propuesta}

La arquitectura del sistema propuesto se basó en el patrón arquitectónico de software Modelo-Vista-Controlador, dicho patrón se utilizó en dos ocasiones dado que, se contempló una aplicación Web y móvil.

Como se mencionó en el párrafo anterior, se consideró una aplicación Web, dicha aplicación se encuentra construida sobre el marco de trabajo Java Server Faces para hacer uso de todas las ventajas que tienen sus herramientas al construir aplicaciones Web.

Por otra parte la aplicación móvil se propone su desarrollo de forma híbrida utilizando el marco de trabajo PhoneGap el cual hace uso de HTML5, JavaScript y CSS para su realización. Su funcionamiento es similar a una página Web dentro del navegador y la interfaz gráfica de usuario se desarrollará con componentes HTML, también permite que el código JavaScript emita llamadas al sistema nativo, por ejemplo para acceder a la libreta de 
direcciones o al sistema de cámara. De esta manera se crea un único proyecto y PhoneGap se encarga de empaquetarlo para diferentes sistemas operativos móviles.

En la Figura 1 se muestra la arquitectura para el sistema propuesto. Cabe mencionar que el discente no podrá acceder al módulo Generador de Feedback en la aplicación Web, solamente a los resultados almacenados en la BD que se obtendrán de dicho módulo y se empleará PhoneGap para el empaquetado de la aplicación móvil.

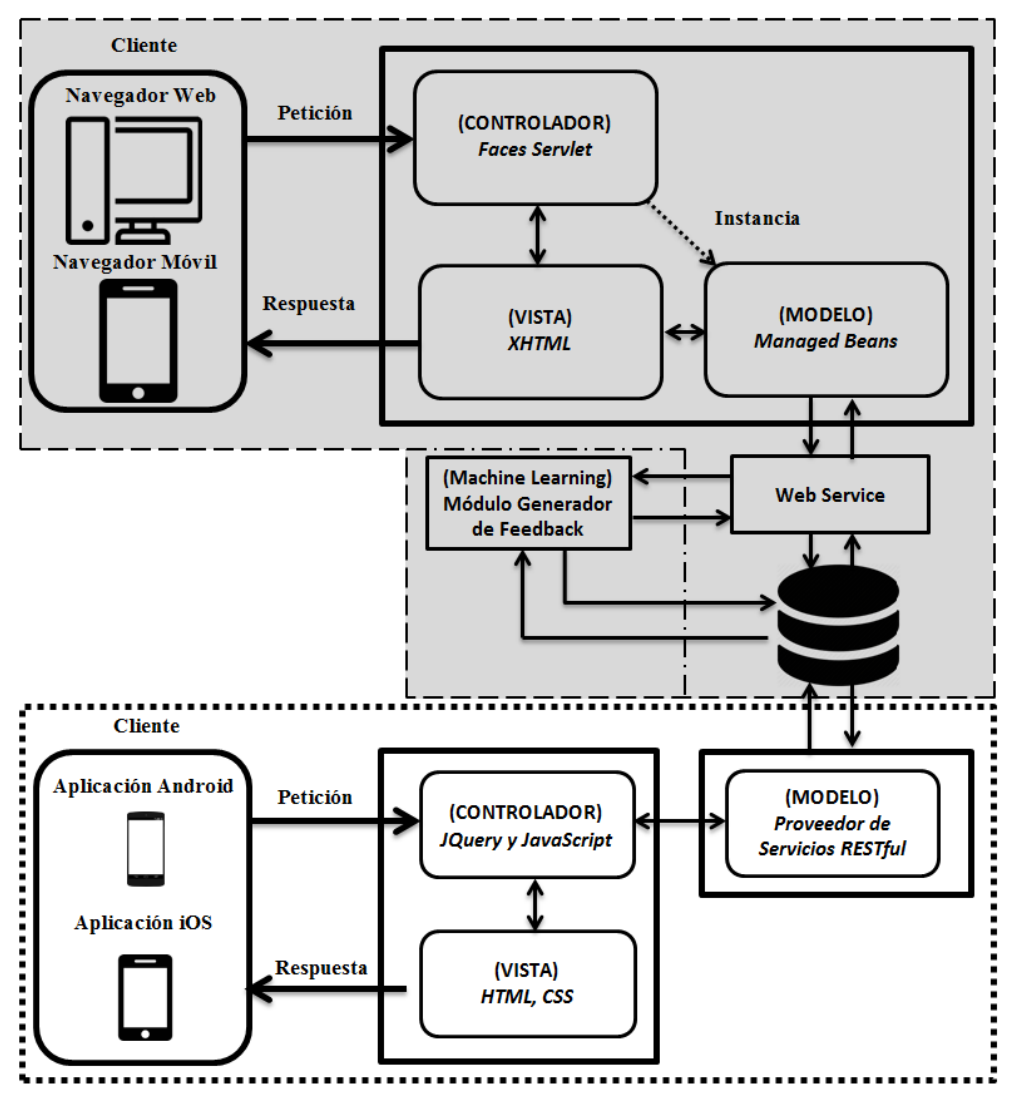

Fig. 1. Patrón arquitectónico MVC aplicado en el sistema propuesto.

\subsection{Modelo-vista-controlador para la plataforma Web}

Como se mencionó en el párrafo anterior, se contempla una aplicación Web, dicha aplicación está construida sobre el marco de trabajo Java Server Faces.

A continuación se ofrece una descripción de la figura 1 explicando la sección remarcada en color gris. 


\subsubsection{Descripciones}

- Cliente: Los clientes para esta parte de la arquitectura fueron pensados para ser un navegador Web convencional o un navegador Web móvil, ambos realizan peticiones de manera independiente al marco de trabajo Java Server Faces solicitando la información que necesiten, el marco reciben las peticiones y las procesa de manera adecuada arrojando un resultado satisfactorio o en su defecto alguna página de error.

- Controlador: Dado que la arquitectura contempla el marco de trabajo Java Server Faces, el componente Faces Servlet funge como controlador recibiendo las peticiones del cliente, el controlador tiene el trabajo de ser el intermediario entre el modelo y la vista, por lo que si el cliente necesita cierta información, el controlador deberá solicitar al modelo dichos datos, instanciando a los JavaBeans necesarios para el proceso y al mismo tiempo preparando la vista para el despliegue de información como respuesta hacia el cliente.

- Modelo: Los beans administrados tienen la tarea de acceder a la información donde quiera que se encuentre sin que el controlador se entere. En este caso el controlador crea instancias del JavaBean necesario para obtener alguna información, de esa manera el JavaBean procede a realizar peticiones al servicio Web basado en tecnología SOAP, este a su vez realiza una petición a la base de datos gestionada en PostgresSQL donde se encuentra almacenada la información, se procesa y se proyecta en la vista para el cliente.

- Vista: La vista son todas aquellas páginas que se despliegan hacia el cliente, desde solicitudes de información, peticiones de otras páginas, errores, etc.

\subsubsection{Web Service y datos}

El servicio Web SOAP se aloja en un servidor de aplicaciones GlassFish a la espera de peticiones por parte del bean administrado, éste contiene todos los diferentes métodos necesarios para cubrir todas las solicitudes que se realicen. Los datos son gestionados por PostgresSQL, donde es almacenada la información.

\subsection{Modelo-vista-controlador para la plataforma Móvil}

Como se mencionó en el punto tres, se contempló una aplicación móvil, dicha aplicación es híbrida por lo que está construida bajo HTML5, JavaScript y CSS.

A continuación se ofrece una descripción de la figura 1 explicando la sección que no está remarcada con color. 


\subsubsection{Descripciones}

- Cliente: El cliente es la propia aplicación móvil, dicha aplicación se compone por páginas HTML5 empaquetadas por el marco de trabajo PhoneGap. La aplicación realiza peticiones por medio del protocolo HTTP solicitando la información que se necesite, procesando de manera adecuada los datos arrojando un resultado satisfactorio o en su defecto alguna página de error.

- Controlador: JQuery y JavaScript fungen como controlador para esta parte, recibiendo las peticiones del cliente. El controlador tiene el trabajo de ser el intermediario entre el modelo y la vista, por lo que si el cliente necesita cierta información, el controlador deberá solicitar al modelo dichos datos para el proceso y a la vez preparando la vista para el despliegue de información como respuesta hacia el cliente.

- Modelo: Como modelo se tiene el proveedor de servicios RESTful, estos tienen la tarea de acceder a la información sin que el controlador se entere. En este caso el controlador solicita información al modelo, éste realiza peticiones a la base de datos gestionada en PostgresSQL donde se encuentra almacenada la información, se procesa y se proyecta en la vista para el cliente.

- Vista: La vista son todas aquellas páginas que se despliegan hacia el cliente, desde solicitudes de información, peticiones a otros enlaces, errores, etc. y que son páginas HTML que se encuentran contenidas como aplicación móvil.

\subsection{Módulo generador de Feedback}

Uno de los módulos principales de la arquitectura propuesta es el denominado Generador de Feedback. Éste se encarga de identificar las dificultades que el discente presenta, usando como entrada los datos recolectados de la interacción del usuario con el sistema, así como las respuestas propias y las de otros usuarios, para ello el acceso al módulo estará disponible únicamente en la interfaz del profesor con el nombre de gráficos el cual desplegará la información pertinente. El módulo tiene la capacidad de agregar herramientas estadísticas o de inteligencia artificial para generar las retroalimentaciones.

Para lograr su cometido, el módulo Generador de Feedback requiere que se almacenen en la base de datos los siguientes datos de cada usuario:

1. Identificador único de usuario (discente y profesor).

2. Tiempos de interacción del usuario con el sistema.

3. Cambios en el estado de la aplicación: Minimizar, Cambio de aplicación, Cerrado de aplicación, Maximizar.

Además, como cualquier otro sistema e-learning que incorpore evaluación, se necesita almacenar los datos relacionados con las preguntas y respuestas. En nuestra propuesta 
actual, se consideran preguntas con respuesta de opción múltiple. Los datos a almacenar son los siguientes:

4. Identificador pregunta.

5. Tema(s) y/o subtema(s) relacionados(s).

6. Pregunta.

7. Respuesta correcta.

8. Distractores.

9. Recomendación tema.

10. Recomendación subtema.

11. Recomendación pregunta.

La relación entre usuarios y preguntas se implementa en otra tabla que contiene los siguientes datos:

12. Identificador usuario.

13. Identificador pregunta.

14. Número de reintentos.

15. Respuesta elegida.

Para probar el módulo propuesto, se implementó una biblioteca propia para cálculos estadísticos en el módulo Generador de Feedback. La estrategia utilizada para generar recomendaciones, toma como base el uso de pruebas de hipótesis (inferencia estadística) para decidir si falla en cada pregunta tiene una dependencia estadísticamente significativa con cierto tema o subtemas relacionados. La prueba de hipótesis elegida para la implementación es la prueba exacta de Fisher, debido a que al inicio los datos recolectados son pocos, y las tablas de frecuencias esperadas contienen valores menores a cinco.

Partiendo del concepto de tutor inteligente, el cual "es un sistema de software que utiliza sistemas inteligentes para asistir al discente que requiere de un tutorizado uno a uno y lo guía en su aprendizaje, adicionalmente posee una representación del conocimiento y una interface que permite la interacción con los discentes para que puedan acceder al mismo" [18].

Para probar el módulo Feeback se propone tomar en cuenta el test exacto de Fisher que es un contraste de hipótesis muy interesante por sus muchas aplicaciones y porque da un útil escenario para aprender la lógica interna de un contraste de hipótesis. Se aplicará en las siguientes situaciones: 1) en la comparación de dos grupos respecto a una variable dicotómica que fue el caso de los géneros de un grupo de 15 discentes. 2) En la valoración de la relación de dos variables cualitativas dicotómicas en temas a evaluar de una materia en específico. Y se procederá a calcular su respectivo porcentaje como en el ejemplo propuesto con datos sintéticos. 
Tabla 2. Propuesta del método de Fisher aplicado al grupo "A".

\begin{tabular}{|c|c|c|c|}
\hline Género & Tema A & Tema B & Total \\
\hline Mujer & 2 & 4 & 6 \\
\hline Hombre & 8 & 1 & 9 \\
\hline Totales & 10 & 5 & 15 \\
\hline
\end{tabular}

Valor-p: 0.0450

Tabla 3. Propuesta del método de Fisher aplicado al grupo "B".

\begin{tabular}{|c|c|c|c|}
\hline Género & Tema A & Tema B & Total \\
\hline Mujer & 3 & 3 & 6 \\
\hline Hombre & 7 & 2 & 9 \\
\hline Totales & 10 & 5 & 15 \\
\hline
\end{tabular}

Valor-p: 0.2398

Tabla 4. Propuesta del método de Fisher aplicado al grupo "C".

\begin{tabular}{|c|c|c|c|}
\hline Género & Tema A & Tema B & Total \\
\hline Mujer & 1 & 5 & 6 \\
\hline Hombre & 9 & 0 & 9 \\
\hline Totales & 10 & 5 & 15 \\
\hline
\end{tabular}

Valor-p: 0.0020

En el ejemplo propuesto se encuentra una dependencia estadísticamente significativa entre las variables analizadas, el sistema elige la retroalimentación para estudiar la sección, pregunta, tema para el discente. Para el profesor, el sistema realiza la retroalimentación basada en las frecuencias de preguntas correctamente e incorrectamente contestadas, indicándole además sobre cuáles temas se encuentran relacionados y cuáles son los más difíciles para los discentes.

Por lo que la interpretación habitual, es que se encuentra dependencia estadística significativa entre las dos variables, si el valor-p es menor a 0.05 . Como puede verse, para el caso de la Tabla 2 y Tabla 4 se encontró dependencia, mientras que para la Tabla 3 no hay dependencia. La retroalimentación en este caso de estudio preliminar y se encuentra en proceso de implementación para la aplicación.

\section{Resultados}

Se muestran algunas pantallas que se han desarrollado para mostrar el estado actual del proyecto, y con ello se espera que la arquitectura propuesta y el módulo de Feedback 
muestren los datos estadísticos de un discente para su futura manipulación en un test de Fisher.

En la Figura 2 se muestra una pantalla donde se despliegan algunas preguntas que forman parte del banco de reactivos para crear evaluaciones.

\begin{tabular}{|c|c|c|c|c|c|c|}
\hline Anco se Preguntst & $x+$ & & & & - & $x$ \\
\hline 4 (1) localhost 000 & thuslolesming Web/taces/prot & sxhtml & c & Buscor & 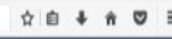 & $\equiv$ 四. \\
\hline MOCHUELO WEB & & & & & & $\infty$ \\
\hline Gestiton & & & estión de Preguntas & & & \\
\hline MMerfi & & & Banco de Preguntas & & & \\
\hline & & & of 1$)=1=$ & & & \\
\hline Graficos & ID Usuario & Tema & Tipo & Pregunta & & \\
\hline & 3 & Sist. e Informática & Abierta & $\begin{array}{l}\text { Cuántas estrellas piensas que } \\
\text { existen en el cosmos }\end{array}$ & Opciones & \\
\hline Cerrar Sesion & 3 & Sist. e Informática & Falso/Nerdadero & $\begin{array}{c}\text { Número de lados de un } \\
\text { triángulo }\end{array}$ & $\begin{array}{l}\text { - Modificar } \\
\text { ×Eliminar }\end{array}$ & \\
\hline & 3 & Sist. e Informática & Multiple & $\begin{array}{l}\text { Selecciona los estados de la } \\
\text { materia }\end{array}$ & Opciones & \\
\hline & 3 & Sist. e Informática & Multiple & Cuál es el valor de Pi & - Opciones & \\
\hline & 3 & Sist. e Intormática & Abierta & $\begin{array}{l}\text { Escribe la definición de una } \\
\text { Intertaz GUI }\end{array}$ & Opciones & \\
\hline & 3 & Sist. e Informática & Multiple & $\begin{array}{l}\text { Lenguaje de Programación } \\
\text { más utilizado actualmente. }\end{array}$ & - Opciones & \\
\hline & 3 & Sist. e Informática & FalsoNerdadero & $\begin{array}{l}\text { ¿Java es el único lenguaje } \\
\text { para crear aplicaciones de } \\
\text { escritorio? }\end{array}$ & - Opciones & \\
\hline & & & f) $=1=$ & & & \\
\hline
\end{tabular}

Fig. 2. Interfaz de usuario para revisar el banco de preguntas.

En la Figura 3 se despliegan algunos datos dentro de la aplicación móvil.

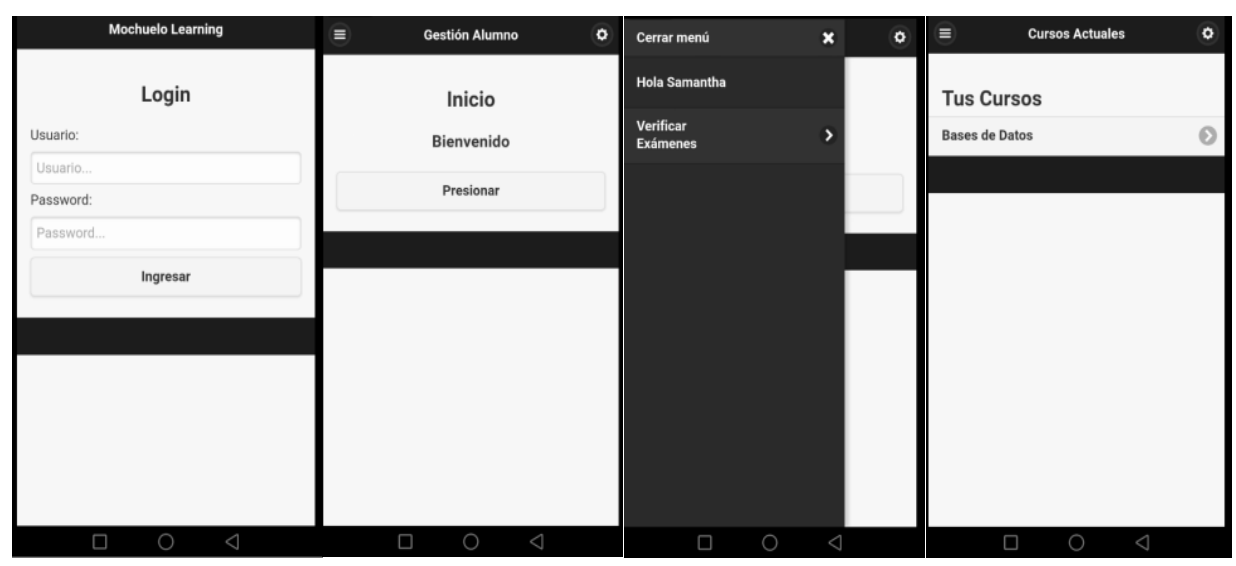

Fig. 3. Consulta de datos en la aplicación móvil.

En la Figura 4 se muestra una pantalla con dos gráficas generadas. 


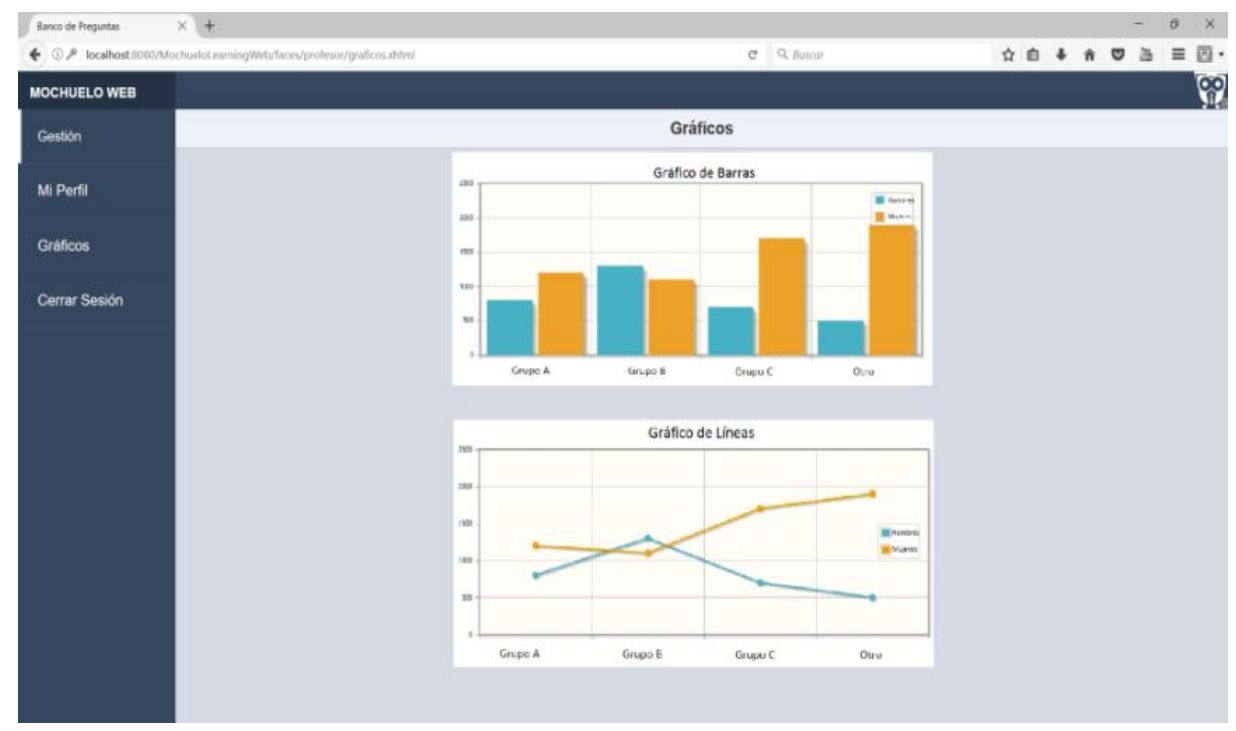

Fig. 4. Gráficas generadas a partir de algunos datos obtenidos.

\section{Conclusiones}

En este artículo presentó una arquitectura escalable de una propuesta de un sistema elearning el cual contiene una aplicación Web y una aplicación móvil, para que se utilicen como apoyo en distintas clases asumiendo los nuevos retos de hoy en día y de esta manera ofrecer cierta retroalimentación basada en el módulo Feedback el cual propondrá información tomada de las calificaciones evaluadas y con esto proporcionar herramientas nuevas empleando la tecnología actual. También se describió de qué forma está compuesta la arquitectura para llevar a cabo este proyecto.

El desarrollar aplicaciones móviles híbridas para el fortalecimiento de formas complementarias de enseñanza es una solución potencial para el mejoramiento continuo del aprendizaje en discentes que cursan sus estudios universitarios o de cualquier nivel superior, además de que el uso de aplicaciones móviles se encuentra en uno de sus puntos más altos, al ser los jóvenes los mayores consumidores de este tipo de medios masivos de comunicación, debido a que día a día se generan miles de aplicaciones disponibles sobre cualquier tema, discusión, materia o interés.

Actualmente, se sigue trabajando en el desarrollo del sistema, principalmente en la implementación de los algoritmos pertinentes del módulo de Feedback como lo es la prueba de Fisher, por lo cual aún no es posible medir el impacto de satisfacción a discentes y maestros. 
Agradecimientos. Los autores agradecen muy gratamente al Congreso Nacional de Ciencia y Tecnología (CONACYT) por el apoyo para realizar este trabajo.

\section{Referencias}

1. Nielsen: The Digital Consumer Report 2014 Nielsen (2014)

2. Sobre penetración actual de dispositivos móviles. Disponible en: http://www. broadbandcommission.org/Documents/reports/bb-annualreport2014.pdf.

3. Isidora, M.: The effects of the intended behavior of students in the use of m-learning. Computers in Human Behavior, Volume 51, Part A, Serbia, pp. 207-215 (2015)

4. Haryadi, D., Joanne, L., Winsen: Mobile learning application based on hybrid mobile application technology running on Android smartphone and Blackberry. In: ICT (Information and Communications Technology) for Smart Society (ICISS), 2013 International Conference on, Jakarta, Indonesia (2013)

5. Bouki, V., Economou, D., Kathrani, P.: Gamification and legal education: A game based application for teaching university law students. In: Interactive Mobile Communication Technologies and Learning (IMCL), 2014 International Conference on, Thessaloniki, Greece (2014)

6. López, J., Cerezo, A., Menéndez, J.: Usage of mobile devices as collaborative tools for education and preparation of official exams. In: International Symposium Consumer Electronics (ISCE), 2015 IEEE International Symposium on, Madrid, Spain (2015)

7. Flores, L., Almario, D., López, J.: Development of an Android App as a learning tool of dynamic systems and automatic control. In: Engineering Mechatronics and Automation (CIIMA), 2014 III International Congress of, Cartagena de Indias, Colombia (2014)

8. Chacon, M., Garita, C.: Mobile Course: Development of a mobile app to access university courses information. In: Computing Conference (CLEI) Conferencia Latinoamericana en Informática, 2013 XXXIX Latin American, Vargas, Venezuela (2013)

9. Hou, J., Zhang, K., Yang, X.: m-learning Course Development and Practice Based on TutorDirected Online Teaching. In: Educational Innovation through Technology (EITT), 2014 International Conference of, Queensland, Australia (2014)

10. Giannakas, F., Kambourakis, G., Gritzalis, S.: CyberAware: A mobile game-based app for cybersecurity education and awareness. In: Interactive Mobile Communication Technologies and Learning (IMCL), 2015 International Conference on, Thessaloniki, Greece (2015)

11. Sarasa, A., Sierra, L.: An app for managing unregulated teaching activities. In: Computers in Education (SIIE) Simposio Internacional de Informática Educativa, 2014, International Symposium on, La Rioja, Spain (2014)

12. Lye, H., Yean, S.: Learning Black Scholes option pricing the fun way via mobile apps. In: Teaching, Assessment and Learning for Engineering (TALE) 2013 IEEE International Conference on, Kuta, Indonesia (2014)

13. Hung, P., Lam, J., Wong, C.: A Study on Using Learning Management System with Mobile App. In: Educational Technology (ISET), 2015 International Symposium on, Wuhan, China (2015) 
14. Martin, S., Gil, R., Cristobal, E.: Middleware for the Development of Context-Aware Applications inside m-learning: Connecting e-learning to the Mobile World. In: Computing in the Global Information Technology, 2009 Fourth International Multi-Conference on, French Riviera, France (2009)

15. Kushik, N., Yevtushenko, N., Evtushenko, T.: Novel machine learning technique for predicting teaching strategy effectiveness. International Journal of Information Management, Russia (2016)

16. Lykourentzou, I.: Dropout prediction in e-learning courses through the combination of machine learning techniques. Computers \& Education, Volume 53, Greece, pp. 950-965 (2009)

17. Huang, X., Zhang, G., Pan, Q.: Research of machine learning methods for student management performance evaluation. In: Information Science and Control Engineering 2012 (ICISCE 2012), International Conference, China (2012)

18. VanLehn, K.: Student Modelling. M. Polson. Foundations of Intelligent Tutoring systems. Hillsdale, N.J. Lawrence Erlbaum Associates, pp. 55-78 (1988) 